\title{
Développement professionnel agentif et collaboratif des enseignants : communautés épistémiques via les réseaux sociaux en temps de crise
}

\author{
Maria Antonieta Impedovo \\ ADEF, Université Aix-Marseille
}

\author{
maria-antonietta.impedovo@univ-amu.fr
}

\begin{abstract}
Résumé
Dans cet exposé, nous explorons les potentialités des réseaux sociaux pour le développement professionnel des enseignants dans une prospective d'agentivité. Le réseautage est une compétence clé dans les carrières professionnelles, soutenant l'apprentissage personnel et professionnel de l'individu, en particulier dans les situations de crise, de transformation personnelle et de changement social. À travers l'observation des groupes de réseaux sociaux (sur Facebook et Reddit) liée à la question du Covid-19 et l'exploration récente de la littérature pédagogique, nous voulons mettre en évidence comment les interactions des enseignants via internet peuvent faciliter le développement professionnel et collaboratif et soutenir un engagement épistémique collectif. La dimension collaborative et agentive exprimée dans l'interaction en ligne conduisant à discuter des implications sur le développement professionnel formel et informel. En fait, les réseaux sociaux peuvent être considérés comme des tiers espaces entre l'apprentissage formel et informel pour soutenir le développement professionnel, apportant également une connotation internationale et interculturelle. Certaines questions sont ouvertes sur les implications de l'engagement en ligne sur la formation initiale et continue des enseignants et sur l'impact des réseaux sociaux pour introduire des innovations dans les pratiques professionnelles et les communautés locales.
\end{abstract}

Mots-clés: Enseignant; Formateurs d'enseignants; Compétences numériques; Réseaux sociaux

\section{Introduction}

La propagation des réseaux sociaux dans le monde est énorme - il y a 7,7 milliards de personnes dans le monde, dont 3,5 milliards en ligne. Cela signifie que les plateformes de médias sociaux sont utilisées par une personne sur trois dans le monde et plus des deux tiers de tous les internautes (Internet World Stats, 2020). Dans le domaine de l'éducation, un nombre croissant d'enseignants ont commencé à s'engager dans des discussions pédagogiques dans des communautés professionnelles en ligne du monde entier (Greenhow et al, 2018 ; Potolia \& Zourou, 2019). Considérant le réseautage comme une compétence clé dans les carrières professionnelles, l'objectif de cet article est une réflexion ouverte sur les potentialités des réseaux sociaux (comme Facebook, Instagram, Twitter -pour citer les plus courants) sur le développement professionnel individuel et collectif des enseignants. 
Cet exposé vise à combler le vide dans notre compréhension de la manière et des raisons pour lesquelles les enseignants utilisent les plateformes numériques en ce qui concerne le développement professionnel formel et informel initial et en cours d'emploi. En effet, si l'utilisation des réseaux sociaux pour l'apprentissage des élèves a suscité plus d'attention et d'échanges, on en sait moins sur l'utilisation de ceux-ci par les enseignants pour leur développement professionnel (Fancera, 2019). En outre, la récente situation de crise mondiale due au Covid-19 a conduit à un changement brusque des pratiques d'enseignement traditionnelles orienté vers de nouvelles pratiques à distance. En plus, en raison du Covid-19, l'enseignant a été isolé et un problème de solitude se pose plus intensément qu'auparavant. De nombreux enseignants se sont donc tournés vers les plateformes numériques, débouchant sur un possible renouvellement de leurs pratiques professionnelles (Ellis et al, 2020).

Deux questions de recherche guident la discussion, toutes deux issues de la littérature pédagogique et d'une observation ethnographique quotidienne des réseaux sociaux des communautés d'enseignants :

1) Pourquoi et comment les enseignants utilisent les réseaux sociaux ?

2) Comment cet engagement influence-t-il le développement professionnel individuel et collectif, aussi en temps de crise?

Pour tenter de répondre, au moins en partie, à ces deux questions, une réflexion est proposée sur la potentialité du réseau social sur le développement professionnel (pour une revue de la littérature : Bruguera, Guitert, \& Romeu, 2019) et l'avantage de soutenir le développement des communautés épistémiques d'enseignants - définie, selon la définition classique de Haas (1992), comme un réseau d'experts fondé sur la connaissance qui définissent ensemble les problèmes auxquels ils sont confrontés, identifient diverses solutions et évaluent les résultats. En fin, cet article vise à soutenir l'importance d'orienter le développement professionnel des enseignants vers une dimension collaborative et agentive marquée en particulier en temps de crise. Le Covid-19 accélère la tendance au changement, ouvre de nouvelles perspectives théoriques et problématiques (Biesta et al, 2020).

À la fin de cet article, des orientations seront discutées sur les implications de l'engagement en ligne des enseignants sur la formation et l'impact de ces échanges pour apporter une innovation durable dans les pratiques professionnelles et les communautés locales.

\section{Développement professionnel collaboratif dans le paysage numérique}

Le développement professionnel des enseignants devient de plus en plus complexe dans la société mondiale en pleine évolution (Littlejohn \& Hood, 2017). En effet, comme c'est également le cas dans de nombreux autres domaines, le développement professionnel est devenu une dimension essentielle pour soutenir la spécialisation croissante et la transformation agile des services. Des défis sont nécessaires pour satisfaire l'apprentissage professionnel en raison des changements sociétaux fluides, de la diffusion de la technologie (Ludvigsen et al, 2019) ou de crises mondiales comme montré par le Covid-19. Selon le modèle d'apprentissage professionnel en trois étapes de Simons et Ruijters (2004), dans ce contexte changeant, les enseignants et les formateurs d'enseignants doivent: 1) élaborer des compétences professionnelles en théorie et en pratique; 2) élargir les connaissances et les idées théoriques en apprenant explicitement de et par la recherche; et 3) externaliser les connaissances pratiques et 
théoriques, ce qui signifie contribuer au développement de la profession et/ou à l'apprentissage en équipe et organisationnel. Le développement de la qualité des enseignants est une question importante, étant donné leur rôle de premier plan et de modèle pour garantir la qualité (Biesta, 2020) et la possible introduction des innovations dans la communauté éducative locale. Le professionnalisme des enseignants émerge comme un espace de tension entre la responsabilité contractuelle et volontaire ; individuel et institutionnel ; responsabilité externe ou interne ; autonomie ou conditionnement (Goodwin, 2020).

Compte tenu de la rapidité et fragilité de la société mondiale interconnectée, comment montrer par la crise due au Covid-19, que la collaboration devient indispensable pour gérer les défis de la globalisation, orienté vers un enrichissement mutuel, pour déclencher des processus de négociation, des objectifs partagés, des ressources et des nouvelles modalités d'action (Goodwin, 2020 ; Van As, 2018). La collaboration (dérivée du monde latin co-labore signifiant travailler ensemble) implique un partage des tâches, une intention explicite de valoriser pour créer quelque chose de nouveau ou de différent à travers un processus collaboratif délibéré et structuré, contrairement à un simple échange d'informations ou exécution d'instructions. Le potentiel de la collaboration est renforcé lorsque cette dernière est médisée par la technique, comme les communications en ligne et les réseaux sociaux (Mora-Ruano et al, 2019 ; Vangrieken et al., 2017).

Les chercheurs en éducation ont donc commencé à explorer l'impact de l'engagement et de la participation active des enseignants dans les médias sociaux et les communautés professionnelles en ligne pour l'apprentissage professionnel (Goodyear et al, 2019). Nous supposons que la discussion des enseignants dans les médias sociaux pourrait être favorable pour découvrir, discuter et suggérer des méthodologies, des outils et des solutions déjà expérimentées par les pairs, soit l'innovation d'introduction. Dans ce qui suit, le concept de communauté de pratique en réseau est exploré comme un outil conceptuel utile à notre discussion.

\section{Communauté de pratique en réseau numérique}

Une perspective pertinente d'analyse conceptuelle du potentiel des sites de réseautage social pour les professionnels est offerte à travers le concept d'une communauté de pratique en réseau (Wenger-Traynor et al., 2015). Il est considéré comme un groupe d'individus qui utilisent des outils numériques - tels que les médias sociaux, les sites $W e b$, les blogs et autres espaces de collaboration en ligne - pour créer des réseaux professionnels, contribuer à l'apprentissage formel / informel et améliorer la croissance personnelle.

La perspective est ancrée dans les perspectives sociales des pratiques (Brown, Collins et Duguid, 1989). Le terme " pratique » représente l'activité qui relie les individus dans leurs réseaux, qui peut également être soutenue par des environnements technologiques (Wenger et al, 2002). Un réseau de pratiques implique un ensemble d'individus connectés par des relations sociales, que ces relations soient fortes ou faibles.

La communauté de pratique en réseau met l'accent sur la centralité des relations, des interactions personnelles et des liens entre les personnes qui partagent des informations, des ressources utiles et contribuent à leur croissance personnelle et professionnelle, personnelle et collective. Si elle est médiatisée par les outils numériques, la communauté en ligne crée, 
soutient et développe des espaces où l'apprentissage pourrait montrer le plus grand potentiel, à la fois pour les personnes et les institutions.

Grâce à l'engagement dans la communauté, le participant devient membre d'une communauté de partage de connaissances orientée vers la construction de nouvelles connaissances et de sens, le partage d'expériences et de solutions à des problèmes réels. Pour rendre compte d'une enquête récente à ce sujet, Eaton et Pasquini (2020) détaillent une étude longitudinale de sept ans sur la façon dont les communautés de pratique en réseau étayent l'apprentissage professionnel et le développement des éducateurs postsecondaires. Les résultats sont centrés sur la nature organique et participative de la communauté, la structure de leadership partagée et la nature bidirectionnelle des relations en ligne et hors ligne telles qu'elles ont été affectées dans les communautés de pratique en réseau.

Dans la section suivante, nous nous intéressons à l'utilisation du réseau social par les enseignants.

\section{Enseignants sur les plateformes de réseaux sociaux}

Généralement, les médias sociaux (comme le blog, le wiki, la plateforme multimédia...) font référence à un large éventail d'application qui permet aux utilisateurs de créer, partager, commenter et discuter du contenu numérique. Les réseaux sociaux analysés sont le système de réseautage social basé sur la conversation. La diffusion du réseau social dans le monde est énorme - il y a 7,7 milliards de personnes dans le monde, dont 3,5 milliards en ligne. Cela signifie que les plateformes de médias sociaux sont utilisées par une personne sur trois dans le monde et plus des deux tiers de tous les internautes (Digital report 2020).

Dans une perspective sociologique, l'usage des médias sociaux répond à l'acquisition du capital social, considéré comme l'ensemble des ressources actuelles ou futures liées à la possession d'un réseau durable de relations de savoir plus ou moins institutionnalisées ou de reconnaissance mutuelle (Bourdieu, 1986), dont la valeur a été reconnue par les enseignants de cette étude. Manca et Ranieri (2017) montrent comment les réseaux sociaux et les sites de réseautage social émergent en tant que lieux où cultiver différentes formes de capital social, comme sous la forme de ponts et de liens. Le capital social de liaison est activé entre les groupes sociaux et le capital social de liaison est activé au sein d'un groupe ou d'une communauté : le premier, qui est généralement composé de liens faibles et occasionnels ; le deuxième avec des liens plus forts et cohérents (Granovetter, 1973). Les liens ont un rôle positif au sein des communautés d'individus dans la mesure où ils facilitent la coordination et la coopération pour un bénéfice mutuel, y compris l'échange de ressources et l'établissement de relations personnelles et professionnelles, avec également des implications pour le bien-être psychologique (Ellison et al, 2011). Les communautés professionnelles en ligne ont un potentiel pour le développement professionnel (Ranieri, 2019). Dans la formation professionnelle, on peut distinguer les réseaux sociaux formels et informels : les premiers sont élaborés par des institutions ayant des objectifs d'apprentissage spécifiques (par exemple, en France, comme Viaeduc, Myriaé, M. @gistere); les réseaux sociaux informels ont été développés avec d'autres finalités principales (comme Twitter, Facebook, Instagram et Snapchat) mais adaptés à la formation, compte tenu de la valeur pour le capital social. Par exemple, les réseaux sociaux comme Facebook, principalement conçus pour la socialisation, sont désormais orientés vers un usage professionnel, un phénomène appelé Facebooking professionnel (Manca \& Ranieri, 2017). Ci-dessous, nous nous concentrons sur les réseaux sociaux informels utilisés par les enseignants. 


\section{Les enseignants sur les réseaux sociaux informels}

La littérature pédagogique suggère que la génération actuelle d'enseignants est de plus en plus engagée dans les médias sociaux informels (Manca \& Ranieri, 2017). Les enseignants du monde entier ont commencé à s'engager dans les réseaux sociaux en ligne, dans différentes matières (Baker-Doyle \& Yoon, 2020 ; Potolia \& Zourou, 2019), lient le potentiel de collaboration avec les potentialités de la technique.

Les enseignants, du primaire aux cycles supérieurs, rejoignent régulièrement des communautés en ligne (comme des groupes sur Facebook ou l'hashtag suivant sur Twitter) pour trouver des ressources pédagogiques appropriées et adaptées, pour explorer les innovations technologiques, pour recevoir des commentaires et pour découvrir des mises à jour sur les nouvelles tendances. Ainsi, les principaux avantages des médias sociaux pour le développement professionnel des enseignants sont le partage de ressources et d'idées (Fox \& Bird, 2017), facilitant l'échange de connaissances (Ranieri, 2019) - conscient du risque possible comme celui découlant de la divulgation de données personnelles. Par exemple, des groupes sur Facebook:

- Le Café pédagogique : groupe publique avec 94,867 participants, présente comment le café pédagogique s'est imposé comme un acteur de l'univers éducatif avec sa douzaine d'éditions (un quotidien, deux mensuels, etc.), 200000 abonnés et près de 800000 visiteurs mensuels ;

- Innovation pédagogique : groupe publique avec 5046 participants, présente comment donner à voir, coopérer, apprendre ensemble, une mise en réseau des innovations pédagogiques dans l'enseignement supérieur francophone.

- Groupe de partage d'expériences, d'entraide et d'innovation pédagogique: groupe publique avec 269 participants, présente comment la création de ce groupe a pour objectif de permettre le partage d'expériences relatives à l'activité des enseignants, quel que soit le type d'activité d'enseignement mené. Ceci ayant pour but l'enrichissement de nos expériences professionnelles. Ce groupe peut aussi être le lieu où certaines problématiques pédagogiques sont soulevées afin d'apporter des pistes de réflexion à l'enseignant. Il est intéressant aussi de proposer de nouvelles idées qui favoriseraient la mise en place d'innovations pédagogiques. Plus largement, ce groupe peut aussi s'ouvrir aux problématiques que peut rencontrer toute personne concernée par la pédagogie et ayant envie d'échanger par rapport à une difficulté rencontrée.

- Enseigner à distance en cycle 2: groupe privé avec 1000 participants, présente comment ce groupe a pour but d'échanger sur les pratiques, les ressources, les mises en œuvre de l'enseignement à distance.

Grâce aux communautés de médias sociaux, les enseignants sont engagés dans des discussions collégiales et peuvent partager des expériences liées à leur profession, avec des interprétations personnelles, ancrées dans les perspectives locales. «L'usage des groupes de discussion sur Facebook représente un moyen efficace pour rejoindre une communauté qui partage un intérêt commun, mais pour en faire une stratégie de développement professionnel efficace, il doit s'inscrire davantage dans le temps, avec des préoccupations liées aux réalités du terrain » (Roy et al, 2020, p. 68). 
L'activité régulière de réseautage en ligne des enseignants des différentes communautés, activement orientée vers le partage des informations, solutions et innovations pédagogique et professionnel, conduit à la consolidation une communauté collaborative épistémique - qui sera expliquée dans la section suivante.

\section{Vers des communautés collaboratives épistémiques en temps de crise}

Dans une communauté épistémique collaborative, les enseignants font l'expérience d'une forme de lien entre l'apprentissage et le partage des connaissances. En ce sens, les enseignants en tant que voix dans les discussions du réseau social expriment une forme d'engagement épistémique, qui est considérée comme les actions tentant d'atteindre des objectifs épistémiques (Elgin, 2013). Ici de suite sont reportés des exemples dans le seul but d'illustrer de nombreuses discussions des enseignants dans le groupe en ligne en Facebook et Reddit. Par exemple, sur la plateforme Reddit certains thèmes vifs sont particulièrement actifs et liés à l'actualité, comme la transformation didactique due à l'introduction des techniques et récemment le changement déclenché par l'état de santé de Covid-19. Dans le deuxième semestre 2020, on a retrouvé, par exemple, la discussion «Enseigner confinés » dans le groupe en langue français "r/France" (https:/www.redditcom/):

\section{Figure 1}

Capture d'écran du groupe Reddit
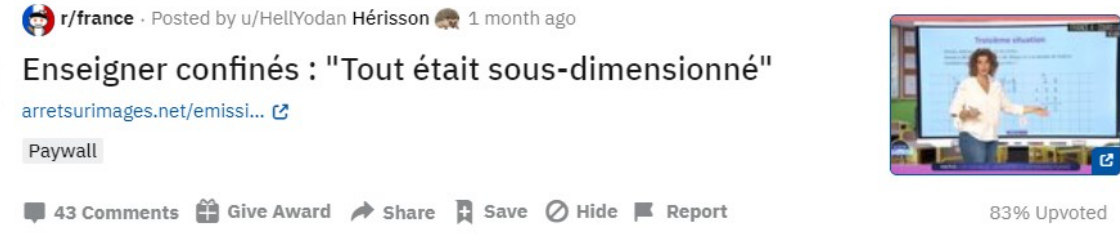

- Post $I$ : «Il évoque les travaux de la sociologue Eszter Hargittai : les élèves feraient face à la" "fracture numérique du second degré" : même s'ils savent se servir des outils numériques, ils ne savent pas toujours les utiliser de manière à apprendre et à se cultiver. Quand je demande à mes élèves d'ouvrir un navigateur, ils ne savent pas ce que c'est. ».

- Post II : «En parlant de "Digital Native" ou "nés sous ère numérique", j'ai retrouvé cet article de 2013 assez pertinent $»$.

- Post III : «Y a quand même un truc que je ne pige pas. On n'est pas censé l'apprendre à l'école justement tout ça ? Enfin, je ne sais pas, mais en cours de techno on avait une partie informatique de base [...]. Forcément si l'école remplit plus son devoir, les élèves ont des lacunes.

Dans les posts considérés, la discussion se concentre sur les tensions qui ont émergé de la gestion du Covid-19, en particulier sur les compétences informatiques des étudiants. Le partage est orienté vers l'échange d'informations (Post $I$ ) et de ressources (Post II) ou engagé dans des pratiques réflexives et critiques pour générer de nouvelles connaissances (Post III). Déjà en 2003, dans le livre L'avenir de la connaissance, Verna Allee souligne l'importance de la connexion sur la collecte d'informations, qui peut être accomplie grâce à des conversations. Ce processus aide à tisser les fils d'une compréhension partagée en de nouvelles synthèses et 
perspectives. On peut donc considérer que, grâce à un engagement actif sur les médias sociaux et dans les communautés professionnelles en ligne, les enseignants peuvent établir des liens et faciliter un apprentissage bénéfique ainsi qu'un partage des connaissances. Dans d'autres communautés ; des enseignantes sur Facebook expriment leurs doutes et leur découragement quant à la gestion de la situation Covid-19 en classe :

- Topic Personnels fragiles ou malades : enfin la circulaire. Post I: « Lol, les élèves dans une salle avec un surveillant et le prof à distance !! Ne y a pas assez de surveillants déjà pour faire tourner les bahuts correctement sans avoir à les réquisitionner pour surveiller les classes qui sont en cours à distance !! Que le gouvernement met des moyens HUMAINS partout pour changer au lieu de réduire toujours et encore les personnels.»

- Topic Covid : Le gouvernement dans ses contradictions. Post II : « Bop, quand je vois les lycéens une fois hors de l'enceinte de l'établissement quitter leurs masques pour s'embrasser et fumer, je me conforte dans l'idée que porter le masque toute la journée ne nous protégera pas ».

Dans ces deux derniers exemples (Post l'and Post II), les enseignants participent aux discussions pour simplement exprimer leur malaise face à la situation à laquelle ils sont confrontés, à la recherche ou à l'offre d'informations et de solutions.

Les enseignants, en tant qu'experts dans une communauté épistémique, sont engagés dans le partage et la construction d'un sens collectif sur leur propre expérience d'enseignement. Ainsi, l'engagement en ligne des enseignants ouvre un accès immédiat et continu à un espace et des savoirs partagés, qui aide l'individu à se connecter avec le collectif et à améliorer potentiellement son développement professionnel en manière agentive. La dimension agentive du développement professionnel des enseignants est explorée dans la section suivante.

\section{La dimension agentive du développement professionnel des enseignants}

L'agentivité enseignante est considérée comme une dimension importante du professionnalisme des enseignants (Deschênes \& Laferrière, 2019), elle permet d'examiner comment les enseignants vivent et prennent des actions dans le monde de l'école en relation avec les pratiques pédagogiques. En plus, l'agentivité (qu'on retrouve traduit aussi comme le pouvoir d'agir et agence ou Human Agency en anglais) est donc liée à ce que les gens font et réalisent dans un contexte spécifique (Biesta \& Tedder, 2006) et à l'expression de sa propre volonté dans les actions qui transforment la réalité. "L'agentivité se manifeste lorsque des personnes formulent des intentions et exécutent des actions volontaires qui vont au-delà des habitudes acceptées et des conditions données de l'activité et de l'organisation dans laquelle elles s'inscrivent, pour ensuite les transformer » (Engeström \& Sannino, 2013, p. 4).

Il doit être compris comme un processus intégré dans le temps présent, influencé par le passé et orienté vers le futur, comme capacité à imaginer des possibilités alternatives. En fait, dans leur modèle écologique, Priestley, Biesta et Robinson (2012) considèrent l'agentivité comme un phénomène qui résulte de l'interaction des efforts individuels, des ressources disponibles et des facteurs contextuels et structurels. Ce concept centralise l'attention sur l'individu (et des groupes) et sur la transformation de la réalité. 
La volonté personnelle (et collective) émerge d'une négociation entre individuel et collectif, voire un déclenchement potentiel des résistances et des tensions (Deschênes \& Laferrière, 2019). La dimension agentive de l'enseignant trouve un espace d'expression dans le partage et la création des ressources numériques, dans la recherche de solutions à mettre en œuvre ultérieurement en classe, dans le soutien des collègues pour une qualité professionnelle partagée, dans la volonté de mettre en œuvre cette dynamique de partage. La co-construction d'un sens d'un développement professionnel collectif devient donc un passage obligé pour une professionnelle de qualité (Stumpf et al, 2020). Le développement professionnel dans un contexte social se nourrit essentiellement des interactions sociales, des débats, des échanges, des confrontations, des partages. Dans le groupe Facebook "le café pédagogique", la publication intitulée "Vaccination : JM Blanquer ne voit pas les enseignants prioritaires » a suscité 578 commentaires et 102 partages. Ainsi, écarter les enseignants des prioritaires a lancé une polémique où le ton a varié entre l'ironie, la satire ou la dramatisation du sujet (" L'éducation ne fait plus partie de la priorité de nos gouvernements successifs »), la discussion s'est tournée aussi vers les statistiques en se basant sur les considérations personnelles et collectives («La vaccination, ça dépasse de loin 1 'éducation nationale et la sphère du privé. Si on veut barrer la route au virus, il faudra un effort collectif»). Certains de ces internautes, réactifs tout au long de la discussion ne cessent de relancer le débat et de répondre aux commentaires ultérieurs en s'impliquant complètement dans le débat.

Un accompagnement formel devient nécessaire pour un véritable développement professionnel avec appropriation des savoirs, des compétences et des réflexions critiques. Pour un véritable développement professionnel, l'apprentissage par la formation formelle est considéré d'un côté comme un levier primordial menant à l'acquisition de savoirs, de compétences et de réflexions (Brennan, 2012). De l'autre côté, la dimension relationnelle, soit avec les collègues ou via les réseaux sociaux sur le Web, permet des apprentissages professionnels grâce aux échanges ayant lieu directement ou sur les médias numériques. Cette discussion informelle représente une excellente occasion de socialiser et d'apprendre à l'intérieur d'une équipe (réelle/virtuelle).

En plus, le retour en classe permet une remise en question critique des implications d'un développement professionnel médiatisé par des ressources sociales, entre épistémologie pratique et agentivité (Boutonnet, 2019). En fait, les contraintes institutionnelles sont plus susceptibles d'être restreintes à l'autonomie, l'innovation et l'autorité du personnel enseignant (Cuban, 1993) en rapport aux réseaux sociaux informels, caractérisés par une dimension volontaire, participative et ludique. Dans la suite, les différents aspects issus de la littérature et de l'observation des réseaux sociaux seront mis en discussion.

\section{Discussion}

L'article discute du fait que le développement professionnel via les réseaux sociaux et les médias sociaux peut être nourri à travers un échange intense d'informations et les liens croissants entre les personnes, les connaissances et les communautés (McIntyre, 2012). Une situation qui devient plus nécessaire en particulier en situation de crise. Pour cela, l'analyse du développement professionnel via les réseaux sociaux peut aider à comprendre l'évolution du développement professionnel et la dynamique de co-construction de savoirs collectifs.

L'apprentissage informel via les réseaux sociaux et les médias sociaux peut combler le vide dans le besoin croissant de qualité du développement professionnel initial et en cours d'emploi des enseignants. La formation formelle des enseignants n'est souvent pas conforme 
aux besoins, aux attentes ou aux contraintes pratiques dans le temps et l'espace. Par exemple, selon les résultats de l'enquête internationale sur l'enseignement et l'apprentissage (TALIS) 2018, la participation au développement professionnel continu des enseignants est nettement plus faible en France (83\%). Ce score est le plus bas de tous les pays participant à TALIS. La barrière à leur participation a augmenté de trois points de pourcentage en France depuis 2013. Parmi les barrières possibles qui pourraient expliquer ces résultats, les enseignants en France rapportent le manque de motivation (47\%), les conflits d'horaire de travail (45\%) ou le manque de temps dû aux responsabilités familiales (45\%), mais aussi l'absence d'offre pertinente (40\%). Les enseignants rapportent que le perfectionnement professionnel fondé sur la collaboration et les approches collaboratives de l'enseignement est parmi les plus efficaces pour eux. De plus, dans les États de l'Organisation de coopération et de développement économiques (OCDE), le développement de compétences avancées en TIC pour l'enseignement est un domaine dans lequel les enseignants disent avoir besoin de plus de formation, à la lumière des transformations technologiques rapides. Pour continuer avec l'exemple du contexte français, l'éducation aux réseaux sociaux et avec les réseaux sociaux fait depuis des années apparaître dans les textes de l'éducation nationale française (Bulletin officiel $n^{\circ} 17 \mathrm{du} 23$ avril 2015 et Bulletin officiel $\mathrm{n}^{\circ}$ $45 \mathrm{du} 9$ mars 2017).

Cette articulation entre la formation formelle et la recherche de solutions informelles de développement professionnel montrent comment les réseaux sociaux jouent le rôle de « tiers espaces » (Bhabha, 2006). Ce terme est utilisé ici pour soutenir l'idée que les réseaux sociaux peuvent être un espace intermédiaire qui forme la frontière entre la formation formelle des enseignants (formation initiale et continue des enseignants, souvent caractérisée par d'importants coûts administratifs et formalités) et les expériences quotidiennes. Les réseaux sociaux dans une dimension intermédiaire joignent les réflexions, les comparaisons critiques et la construction de ressources, alliées au plaisir des échanges. La volonté personnelle du partage et de l'entraide est le point de départ de la dynamique d'apprentissage personnel et d'un sens d'un développement professionnel collectif (Stumpf et al, 2020). La construction de significations engagées dans un processus de co-élaboration de connaissances (Laferrière, 2020), dans les interactions entre pairs, conduit à façonner des expériences d'enseignement de qualité.

De plus, on peut considérer que les enseignants connectés avec d'autres via le web ont l'opportunité d'être confrontés à des expériences plus équitables et moins hiérarchiques dans les institutions professionnelles ou d'apprentissages locales. En revanche, les enseignants doivent être conscients des problèmes de confidentialité, de privacy et le dilemme éthique qui peuvent survenir facilement en partageant des informations personnelles ou des informations sur leurs élèves (Goodyear, 2017).

L'échange en ligne permettra aux enseignants d'incorporer de nouvelles perspectives et compétences nécessaires pour faire face aux nouveaux défis (Nerantzi, 2018). Le réseautage social expose l'enseignant à des communautés élargies est particulièrement crucial en période de crise, comme le montrait l'actualité du Covid-19, où les émotions stressantes, la solitude et la désinformation pourraient compromettre la qualité de la vie personnelle et professionnelle (Ellis, Steadman, \& Mao, 2020).

Enfin, un échange d'expériences d'enseignement via des plateformes sociales en ligne contribuera à un professionnalisme partagé accru, également à connotation internationale et interculturelle (Goodwin, 2020). Ainsi, les enseignants peuvent se sentir intégrés à une 
communauté mondiale étendue avec les mêmes objectifs de qualité et même défaite : par exemple, pendant la crise due au Covid-19, le problème de l'enseignement à distance était une situation d'urgence partagée à niveau mondial.

Comprendre comment maximiser le potentiel de connexion via les réseaux sociaux avec d'autres communautés d'enseignants reste un défi de taille. Les recherches futures se concentreront sur le développement théorique et pragmatique de la manière de soutenir un développement professionnel agentif et collaboratif qui prend en compte à la fois les implications de la participation dans un espace de réseau social informel et la participation dans le contexte scolaire et institutionnel formel. L'enjeu devient aussi celui d'une articulation entre une approche sociotechnique et l'activité individuelle et collective en formation professionnelle (Albero et al, 2019), s'orientant vers la conception formelle d'environnements de qualité dans la formation des enseignants, partant précisément du potentiel et des limites des réseaux sociaux informels.

Pour conclure, les réflexions développées dans cet article prospectif vont dans le sens de la promotion d'une ouverture accrue et d'une collaboration entre la personne et les institutions, reliant l'apprentissage formel et informel, notamment dans les situations de crise, de transaction et de changement.

\section{Références}

Albero, B., Guérin, J., \& Watteau, B. (2019). Comprendre la relation entre influences de l'environnement et activité : questionnements théoriques et enjeux praxéologiques. Savoirs (1), 103-124.

Allee, V. (2003). The future of knowledge: Increasing prosperity through value networks. Routledge.

Baker-Doyle, K. J., \& Yoon, S. A. (2020). The social side of teacher education: Implications of social network research for the design of professional development. International Journal of Educational Research, 101, 101563. https://doi.org/S0883035519327193

Bhabha, H. K., \& Rutherford, J. (2006). Le tiers-espace. Multitudes, (3), 95-107.

Biesta, G. (2020). What constitutes the good of education? Reflections on the possibility of educational critique. Educational Philosophy and Theory, 1-5. https://doi.org/10.1080/00131857.2020.1723468

Biesta, G., Takayama, K., Kettle, M., \& Heimans, S. (2020). Call for papers: A new agenda for teacher education research. Asia-Pacific Journal of Teacher Education, 1-3. https://doi.org/10.1080/1359866X.2020.1818484

Bourdieu, P. (1986). Habitus, code et codification. Actes de la recherche en sciences sociales, 64(1), 40-44.

Brown, J.S., Collins, A., \& Duguid, P. (1989). Situated cognition and the culture of learning. Educational Researcher, 18(1), 32-42.

Brennan, K. (2012). Best of both worlds: Issues of structure and agency in computational creation, in and out of school (Ph.D. Thesis). Massachusetts Institute of Technology, Cambridge, MA.

Bruguera, C., Guitert, M., \& Romeu, T. (2019). Social media and professional development: a systematic review. Research in Learning Technology, 27. https://doi.org/10.25304/rlt.v27.2286

Boutonnet, V. (2019). Les pratiques d'enseignement de futures enseignantes et futurs enseignants d'histoire-géographie du secondaire au Québec : épistémologie pratique et 
agentivité. Éducation et francophonie, 47(2), 83-101.

https://doi.org/10.7202/1066449ar

Cuban, L. (1993). How teachers taught: Constancy and change in american classrooms. New York: Teachers College Press.

Denouel J. (2019). D'une approche sociocritique à une approche sociotechnique critique des usages numériques en éducation. Formation et profession, 27, 3, 36-48. https://doi.org/10.18162/fp.2019.483

Deschênes, M., \& Laferrière, T. (2019). Le codesign d'une plateforme numérique fondé sur des principes au service de l'agentivité des enseignantes et des enseignants en contexte de développement professionnel. La revue canadienne de l'apprentissage et de la technologie, 45(1), 1-20. https://doi.org/10.21432/cjlt27798

Kemp, S. (2020). Digital 2020 : 3.8 billion people use social media, Hootsuite. https://wearesocial.com/digital-2020

Eaton, P. W., \& Pasquini, L. A. (2020). Networked practices in higher education: A netnography of the\# acadv chat community. The Internet and Higher Education, 45. https://doi.org/S1096751619304415

Ellis, V., Steadman, S., \& Mao, Q. (2020). 'Come to a screeching halt': Can change in teacher education during the COVID-19 pandemic be seen as innovation? European Journal of Teacher Education, 43(4), 559-572. https://doi.org/10.1080/02619768.2020.1821186

Ellison, N. B., Steinfield, C., \& Lampe, C. (2011). Connection strategies: Social capital implications of Facebook-enabled communication practices. New media \& society, 13(6), 873-892. https://doi.org/10.1177/1461444810385389

Elgin, C. Z. (2013). Epistemic agency. Theory and Research in Education, 11, 135- 140.

Engestrom, Y, Sannino, A. (2013). La volition et l'agentivité transformatrice : perspective théorique de l'activité. Revue internationale du CRIRES : innover dans la tradition de Vygotsky, 1(1), 4-19.

Fancera, S. F. (2019). School leadership for professional development: the role of social media and networks. Professional Development in Education. https://doi.org/10.1080/19415257.2019.1629615

Fox, A., \& Bird, T. (2017). \# any use? What do we know about how teachers and doctors learn through social media use? Qwerty-Open and Interdisciplinary Journal of Technology, Culture and Education, 12(2), 64-87.

Goodyear, V. A. (2017). Social media,apps and wearable technologies: navigating ethical dilemmas and procedures. Qualitative Research in Sport, Exercise and Health, 17 (3), 285-302. https://doi.org/10.1080/2159676X.2017.1303790

Goodyear, V. A., Parker, M., \& Casey, A. (2019). Social media and teacher professional learning communities. Physical Educationand Sport Pedagogy, 24(5), 421-433. https://doi.org/10.1080/17408989.2019.1617263

Goodwin Lin, A. (2020). Teaching standards, globalisation, and conceptions of teacher professionalism. European Journal of Teacher Education. DOI: 10.1080/02619768.2020.1833855.

Granovetter, M. S. (1973). The strength of weak ties. American journal of sociology, 78(6), 1360-1380.

Greenhow, C., Campbell, D. Galvin, S. Askari, E. (2018). Social Media in Teacher Professional Development: A Literature Review. https://www.learntechlib.org/primary/p/182975/. 
Haas, P. (1992). Introduction: epistemic communities and international policy coordination. International Organization. Cambridge Journals. 46 (1), 1-35. https://www.jstor.org/stable/2706951

Internet World Stats, 2020. https://www.internetworldstats.com/stats.htm.

Laferrière, T. (2020). La recherche en partenariat pour l'enseignement d'hier à demain. Revue hybride de l'éducation, 4(1), 94-115. https://doi.org/10.1522/rhe.v4i1.974

Littlejohn, A., \& Hood, N. (2017). How educators build knowledge and expand their practice: The case of open education resources. British Journal of Educational Technology, 48(2), 499-510. https://doi.org/10.1111/bjet.12438

Ludvigsen, S. R., Lund, A., Rasmussen, I., \& Säljö, R. (Eds.). (2010). Learning across sites: New tools, infrastructures and practices. Routledge.

Manca, S., \& Ranieri, M. (2017). Implications of social network sites for teaching and learning. Where we are and where we want to go. Education and Information Technologies, 22(2), 605-622. https://doi.org/10.1007/s10639-015-9429-x

McIntyre, S. (2012). Exploring a Rhizomic Model for the Design and Dissemination of Professional Development in Online Teaching. ICEL, 492-501.

Mora-Ruano, J. G., Heine, J. H., \& Gebhardt, M. (2019). Does Teacher Collaboration Improve Student Achievement? Analysis of the German PISA 2012 Sample. Frontiers in Education, 4, 85. https://doi.org/10.3389/feduc.2019.00085

Nerantzi, C. (2019). The design of an empirical cross-boundary collaborative open learning framework. Open Praxis, 10(4), 325-341. https://doi.org/10.5944/openpraxis.10.4.907

Potolia, A., \& Zourou, K. (2019). Approches réflexives sur l'accompagnement pédagogique au sein d'une communauté web 2.0 d'apprenants de langues. Quels apports et limites pour les enseignants de langue ? Distances et médiations des savoirs. Distance and Mediation of Knowledge, (26). https://doi.org/10.4000/dms.3639

Priestley, M., Biesta, G. J. J. \& Robinson, S. (2012). Understanding teacher agency: The importance of relationships. Annual Meeting of the American Educational Research Association, Vancouver, Canada, 13-17 April 2012. http://www.stir.ac.uk/media/schools/education/documents/teacheragency /Teacher\%20agency_AERA\%20paper_final.pdf

Ranieri, M. (2019). Professional development in the digital age. Benefits and constraints of social media for lifelong learning. Form@re, 19(2), 178-192. ISSN 1825-7321.

Roy, N., Gruslin, É., \& Poellhuber, B. (2020). Le développement professionnel au postsecondaire à l'ère du numérique. Revue internationale des technologies en pédagogie universitaire, 17(1), 63-75. https://doi.org/10.18162/ritpu-2020-v17n1-13

Simons, P. R. J., \& Ruijters, M. C. (2004). Learning professionals: Towards an integrated model. In Professional Learning: Gaps and transitions on the way from novice to expert (pp. 207-229). Springer, Dordrecht.

Stumpf, A., Meia, J. S., \& Garessus, P. A. (2020). Co-construire le sens d'un développement professionnel collectif : un passage obligé. Formation et profession : revue scientifique internationale en éducation, 28(1), 135-138. URI:

https://id.erudit.org/iderudit/1071893ar

TALIS (2018). Results. Teachers and School Leaders as Lifelong Learners. http://www.oecd.org/education/talis-2018-results-volume-i-1d0bc92a-en.htm

Van As, F. (2018). Communities of practice as a tool for continuing professional development of technology teachers' professional knowledge. International Journal of Technology and Design Education, 28(2), 417-430. https://doi.org/10.1007/s10798-017-9401-8

Vangrieken, K., Meredith, C., Packer, T., \& Kyndt, E. (2017). Teacher communities as a context for professional development: A systematic review. Teaching and teacher education, 61, 47-59. https://doi.org/S0742051X16304681 
Revue internationale du CRIRES : innover dans la tradition de Vygotsky (2021), vol 5, no 1

Wenger, E., McDermott, R. A., \& Snyder, W. (2002). Cultivating communities of practice: A guide to managing knowledge. Harvard Business Press.

Wenger-Traynor, E., \& Fenton-O'Creevy, H. S., Kubiak, C. and Wenger-Traynor, B. (2015). Learning in Landscapes of Practice: Boundaries, Identity and Knowledgeability. Practice-Based Learning. 